\title{
Photodisintegration of three- and four- nucleon systems*
}

\author{
$\underline{\text { W. Sandhas }}^{\mathrm{a}}$, W. Schadow ${ }^{\mathrm{a}}$, G. Ellerkmann ${ }^{\dagger}$, L. L. Howell ${ }^{\mathrm{b}}$, and S. A. Sofianos ${ }^{\mathrm{b}}$ \\ aPhysikalisches Institut der Universität Bonn, Endenicher Allee 11-13, D-53115 Bonn, \\ Germany

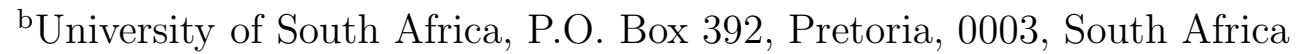 \\ Three- and four-nucleon photodisintegration processes are quite efficiently treated by \\ means of effective two-body integral equations in momentum space. We recall some as- \\ pects of their derivation, present previous and most recent results obtained within this \\ framework, and discuss general features, trends and effects observed in these investiga- \\ tions: At low energies final-state interaction plays an important role. Even more pro- \\ nounced is the effect of meson exchange currents. A considerable potential dependence \\ shows up in the low-energy peak region. The different peak heights are found to be \\ closely correlated with the corresponding binding energies. Above the peak region only \\ the difference between potentials with or without $p$-wave contributions remains relevant. \\ In the differential cross sections the electric quadrupole contributions have to be taken \\ into account. The remarkable agreement between theory and experiment in $p$ - $d$ radiative \\ capture is achieved only when incorporating this contribution, together with most of the \\ above-mentioned effects. In the final part of this report we briefly review also methods \\ developed, and results achieved in three- and four- nucleon electrodisintegration. We, in \\ particular, compare them with a recent access to this problem, based on the construction \\ of nucleon-nucleus potentials via Marchenko inversion theory.
}

\section{THREE-BODY PROCESSES}

Starting form the momentum space formulation of three-nucleon collision processes, we sketch the transition to the corresponding photodisintegration equations. Results obtained within this approach are presented, general trends, sensitivities, and correlations are discussed.

\subsection{Deuteron photodisintegration}

The characteristic features of the momentum-space approach are most easily described in the two-body case. There, the scattering amplitude is given by the on-shell restriction $\left(p^{2}=p^{2}\right)$ of the $T$-matrix

$$
T\left(\vec{p}^{\prime}, \vec{p}\right)=\left\langle\vec{p}^{\prime}|T| \vec{p}\right\rangle={ }^{(-)}\left\langle\vec{p}^{\prime}|V| \vec{p}\right\rangle,
$$

*Invited talk at the XVth International Conference on Few-Body Problems in Physics (22-26 July 1997, Groningen, The Netherlands)

†The work of W. S, W. Sch., and G. E. was supported by the Deutsche Forschungsgemeinschaft 
where $|\vec{p}\rangle$ represents the incoming plane-wave state, and ${ }^{(-)}\left\langle\vec{p}^{\prime}\right|$ the outgoing scattering state. The T-operator in (1.1) satisfies the Lippmann-Schwinger (LS) equation

$$
T=V+V G_{0} T
$$

Being closely related to the physical amplitude, this operator is well- defined in momentum space also off the energy shell $\left(p^{\prime 2} \neq p^{2}\right)$, and the same holds true for the momentum representation of (1.2),

$$
T\left(\vec{p}^{\prime}, \vec{p}\right)=V\left(\vec{p}^{\prime}, \vec{p}\right)+\int d^{3} p^{\prime \prime} V\left(\vec{p}^{\prime}, \vec{p}^{\prime \prime}\right) G_{0}\left(p^{\prime \prime}\right) T\left(\vec{p}^{\prime \prime}, \vec{p}\right)
$$

There is only a singularity in the Green function $G_{0}\left(p^{\prime \prime}\right)=\left(p^{2} / 2 \mu+i \epsilon-p^{\prime \prime 2} / 2 \mu\right)^{-1}$ at the on-shell point $p^{\prime \prime 2}=p^{2}$, which however is easily taken care of. A useful way of doing this is provided by the $K$-matrix approach or, more efficiently, by the $W$-matrix method. We mention this method since it yields a particularly convenient representation of the two-body input to the three- and four-body treatments discussed in the following.

Analogously to (1.1), the amplitude for the photodisintegration of the deuteron $\left|\psi_{d}\right\rangle$ is given by

$$
M(\vec{p})={ }^{(-)}\left\langle\vec{p}\left|H_{e m}\right| \psi_{d}\right\rangle=\left\langle\vec{p}\left|\left(1+T G_{0}\right) H_{e m}\right| \psi_{d}\right\rangle,
$$

where $H_{e m}$ denotes the electromagnetic operator. Here we have made use of the fact that the momentum state $\langle\vec{p}|$ is mapped upon the scattering state ${ }^{(-)}\langle\vec{p}|$ via the adjoint Møller operator $\left(1+T G_{0}\right)$. This representation shows that the amplitude (1.4) is composed of a plane wave (Born) term

$$
B(\vec{p})=\left\langle\vec{p}\left|H_{e m}\right| \psi_{d}\right\rangle
$$

and a further contribution $\left\langle\vec{p}\left|T G_{0} H_{e m}\right| \psi_{d}\right\rangle$ which, via $T$, takes into account the final-state interaction(FSI). Inserting in this expression the LS equation (1.2), we obtain for $M(\vec{p})$ the integral equation

$$
M(\vec{p})=B(\vec{p})+\int d^{3} p^{\prime} V\left(\vec{p}, \vec{p}^{\prime}\right) G_{0}\left(\vec{p}^{\prime}\right) M\left(\vec{p}^{\prime}\right) .
$$

Equations (1.3) and (1.6) are evidently of the same structure. Their kernels $V G_{0}$, i.e., their most relevant ingredients, are identical. But, the nuclear Born approximation $V\left(\vec{p}, \vec{p}^{\prime}\right)$ in the inhomogeneity of (1.3) is replaced in (1.6) by the photonuclear Born term (1.5). In other words, with this comparatively simple replacement, any working NN collision program based on (1.3) can immediately be applied to deuteron photodisintegration.

The present derivation serves primarily to exhibit structural aspects, which are typical also for the corresponding three- and four-nucleon generalizations. It, moreover, provides a quite efficient numerical tool, characterized by considerable stability, as emphasized in the context of (1.3). But, of course, in the two-body case there are numerous alternative techniques at our disposal (for a comprehensive review see [1]). In the three- or four-body case, however, the generalization of (1.6) represents one of the most efficient standard techniques. 
In electric dipole (E1) approximation the amplitude (1.4) is given by

$$
M(\vec{p})=-\frac{e}{2 \mu}{ }^{(-)}\left\langle\vec{p}|\hat{\epsilon} \cdot \vec{P}| \psi_{d}\right\rangle=-\frac{i e}{2}{ }^{(-)}\left\langle\vec{p}\left|\hat{\epsilon} \cdot\left[H_{0}, \vec{X}\right]\right| \psi_{d}\right\rangle
$$

where $\hat{\epsilon}$ denotes the polarization vector of the incident photon. Replacing in the commutator the free Hamiltonian $H_{0}=P^{2} / 2 \mu$ by the full Hamiltonian $H=H_{0}+V$, this expression goes over into

$$
M(\vec{p})=-\frac{i e}{2}\left(E_{f}-E_{i}\right)^{(-)}\left\langle\vec{p}|\hat{\epsilon} \cdot \vec{X}| \psi_{d}\right\rangle
$$

with $\left(E_{f}-E_{i}\right)=E_{\gamma}$ being the difference of the final energy $E_{f}=p^{2} / \mu$ and the initial deuteron energy $E_{i}=E_{d}$, i.e., the energy $E_{\gamma}$ of the photon.

For local potentials, which commute with $\vec{X}$, the transition to (1.8) is only another, in some approaches technically more convenient way of writing the amplitude (1.7). For the typically non-local nuclear potentials, the replacement of (1.7) by (1.8) leads in general to quite different results. But, according to Siegert's theorem [2], it is just this replacement, which takes into account the effect of meson exchange currents (MEC) in the nucleus. Siegert's argumentation involves, of course, an approximation. But, at least in the deuteron case it has been shown that the results obtained in this way fully agree with calculations based on a Hamiltonian which includes the MEC contributions explicitly [1]. In the three- and four-body cases the validity of Siegert's theorem is usually taken for granted, an assumption which would need a similar verification as in the two-body case.

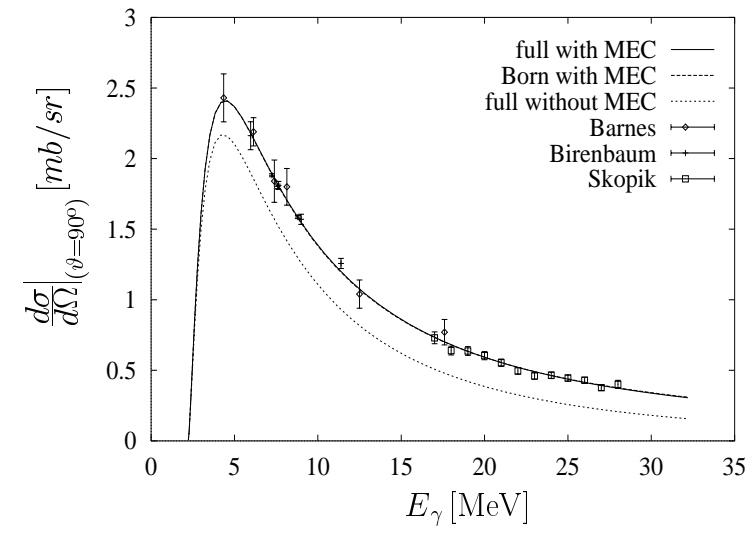

Figure 1. Role of meson exchange currents and final-state interaction. The experimental data are from [4 [6].

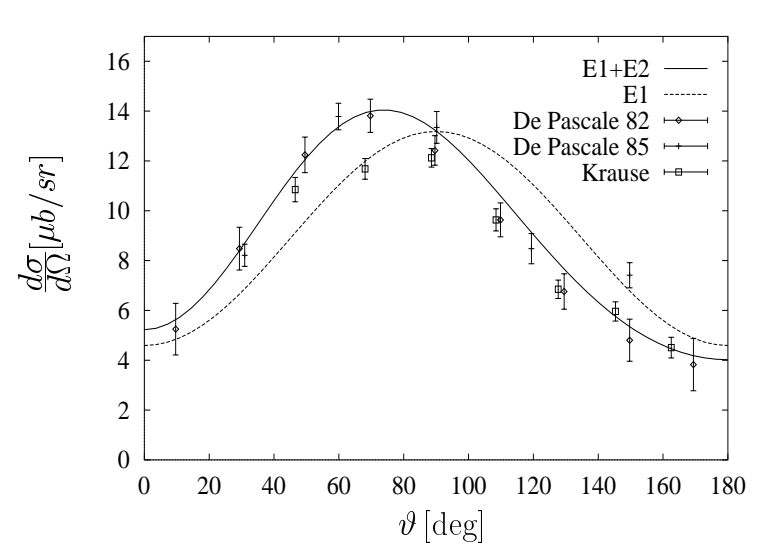

Figure 2. Differential cross section for deuteron photodisintegration with the Bonn $B$ potential in $E 1$ and $E 1+E 2$ approximation at $E_{\gamma}=60 \mathrm{MeV}$. The experimental data are from [7,8].

Fig. 11 shows the differential cross section at $\vartheta=90^{\circ}$ in $E 1$ approximation, obtained for the Paris potential by solving (1.6). The full solution is almost identical with the Born 
result, i.e., the FSI is practically negligible in the energy region considered. But there is a clear MEC effect. Fig. 2 shows the differential cross section in $E 1$ approximation, and with inclusion of the electric quadrupole $(E 2)$ contribution. It should be mentioned that these results are in complete agreement with an independent solution in Ref. [3].

One of the main questions being asked in the following is, whether similar or quite different FSI, MEC or E2 effects occur in the corresponding three- and four-nucleon processes.

\subsection{Three-body equations}

In the three-body case the $T$-matrix for rearrangement processes $\alpha+(\beta \gamma) \rightarrow \beta+(\alpha \gamma)$ is given by

$$
T_{\beta m, \alpha n}\left(\vec{q}_{\beta}^{\prime}, \vec{q}_{\alpha}\right)=\left\langle\vec{q}_{\beta}^{\prime}\left|\left\langle\psi_{\beta m}\left|U_{\beta \alpha}\right| \psi_{\alpha n}\right\rangle\right| \vec{q}_{\alpha}\right\rangle
$$

where $\vec{q}_{\alpha}$ denotes the momentum of particle $\alpha$ relative to the bound state $\left|\psi_{\alpha n}\right\rangle$ of particles $\beta$ and $\gamma$. Thus, due to the various initial and final configurations $(\alpha, \beta=1,2$ or 3$)$, we have, instead of a single $T$-operator, a set of $3 \times 3$ transition operators. Correspondingly, the single LS operator identity (1.2) is replaced by the coupled set of Faddeev-type AltGrassberger-Sandhas (AGS) equations [9]

$$
U_{\beta \alpha}=\left(1-\delta_{\beta \alpha}\right) G_{0}^{-1}+\sum_{\gamma \neq \beta} T_{\gamma} G_{0} U_{\gamma \alpha}
$$

These equations contain in their kernel the three subsystem $T$-operators $T_{\gamma}=T_{\alpha \beta}$. In other words, they represent in a most condensed way the relation between two-body input and three-body output. Of particular importance is that, like (1.2), they are well-defined in momentum space, and thus amenable to the particularly accurate momentum- space methods.

As three-body operators, the $U_{\beta \alpha}$ act on the momentum state $\left|\vec{q}_{\alpha}\right\rangle$ of particle $\alpha$ relative to the $(\beta \gamma)$ system, and on the momentum state $\left|\vec{p}_{\alpha}\right\rangle$ within this subsystem. In momentum representation the AGS equations, hence, are two-dimensional after partial wave decomposition. They were used successfully in this direct form which, however, needs a considerable numerical effort. From the very beginning applications of (1.10), therefore, made use of the most typical aspect of Faddeev-type equations, the occurrence of $T_{\gamma}$ in their kernel. Representing these operators in separable form,

$$
T_{\gamma}=\sum_{n m}\left|\chi_{\gamma n}\right\rangle t_{\gamma, n m}\left\langle\bar{\chi}_{\gamma m}\right|
$$

Eq. (1.10) reduces to a set of effective two-body equations of LS form which, in matrix notation, reads

$$
\mathcal{T}\left(\vec{q}^{\prime}, \vec{q}\right)=\mathcal{V}\left(\vec{q}^{\prime}, \vec{q}\right)+\int d^{3} q^{\prime \prime} \mathcal{V}\left(\vec{q}^{\prime} \vec{q}^{\prime \prime}\right) \mathcal{G}_{0}\left(q^{\prime \prime}\right) \mathcal{T}\left(\vec{q}^{\prime \prime} \vec{q}\right)
$$

Here, the matrix elements of $\mathcal{T}$ are off-shell extensions of (1.9), the elements of $\mathcal{V}$ are exchange potentials

$$
\mathcal{V}_{\beta m, \alpha n}\left(\vec{q}_{\beta}^{\prime}, \vec{q}_{\alpha}\right)=\left(1-\delta_{\beta \alpha}\right)\left\langle\vec{q}_{\beta}^{\prime}\left|\left\langle\bar{\chi}_{\beta m}\left|G_{0}\right| \chi_{\alpha n}\right\rangle\right| \vec{q}_{\alpha}\right\rangle
$$


and the free Green function is given by

$$
\mathcal{G}_{0 ; \beta m, \alpha n}=\delta_{\beta \alpha} t_{\alpha, n m}
$$

After this reduction, the resulting matrix LS equation (1.12) is, of course, to be antisymmetrized. The analogy of (1.3) and (1.12) indicates that also the photodisintegration equation (1.6) has its counterpart in the present case. A procedure, closely related to the steps leading to (1.6), in fact, yields for the two-fragment photodisintegration of the triton, $\gamma+t \rightarrow n+d$, the effective two-body equation

$$
\mathcal{M}(\vec{q})=\mathcal{B}(\vec{q})+\int d^{3} q^{\prime} \mathcal{V}\left(\vec{q}, \vec{q}^{\prime}\right) \mathcal{G}_{0}\left(\vec{q}^{\prime}\right) \mathcal{M}\left(\vec{q}^{\prime}\right)
$$

Here, $\mathcal{M}(\vec{q})$ and $\mathcal{B}(\vec{q})$ are off-shell extensions of the full photodisintegration amplitude

$$
M(\vec{q})={ }^{(-)}\left\langle\vec{q} ; \psi_{d}\left|H_{e m}\right| \psi_{t}\right\rangle
$$

and of the corresponding plane wave (Born) approximation

$$
B(\vec{q})=\langle\vec{q}|\left\langle\psi_{d}\left|H_{e m}\right| \psi_{t}\right\rangle
$$

with $\vec{q}$ the relative momentum between the outgoing nucleon and the deuteron, and $\left|\psi_{t}\right\rangle$ the triton wave function.

The decisive advantage of this formalism is that the appropriate construction of $\mathcal{V}$ and $\mathcal{G}_{0}$, including tests of accuracy, is well known from the purely nuclear case, and has not to be repeated. What remains to be done, when going over to the photonuclear equation $(1.15)$, is the consistent determination of the off-shell Born term $\mathcal{B}(\vec{q})$. For details we refer to the papers quoted in the next section.

Let us make some remarks on the derivation of (1.15). In Eq.(1.4) it is the adjoint Møller operator $\left(1+T G_{0}\right)$ which maps $\langle\vec{p}|$ onto ${ }^{(-)}\langle\vec{p}|$. Analogously, we have in the present case

$$
M\left(\vec{q}_{\beta}\right)=\left\langle\vec{q}_{\beta}\right|\left\langle\psi_{d}\left|\left(\delta_{\beta \alpha}+U_{\beta a} G_{\alpha}\right) H_{e m}\right| \psi_{t}\right\rangle
$$

with $G_{\alpha}=\left(E_{f}+i \epsilon-H_{0}-V_{\alpha}\right)^{-1}$ the channel resolvent of the $\alpha=(\beta \gamma)$ subsystem. Proceeding in strict analogy to the derivation of (1.6), i.e., inserting here, instead of the LS equation, the AGS equations with $T_{\gamma}$ being chosen according to (1.11), we end up with an effective two-body equation of the form (1.15) with the matrix elements of the inhomogeneity being given by

$$
\mathcal{B}_{n}\left(\vec{q}_{\beta}\right)=\left\langle\vec{q}_{\beta}\right|\left\langle\chi_{\beta m}\left|G_{0} H_{e m}\right| \psi_{t}\right\rangle
$$

We, moreover, mention that consistently with the approximation (1.11) the triton wave function may be determined by solving the homogeneous counterpart of equation (1.12). For details we refer to 10 12].

Three-body photonuclear integral equations were first derived within the Faddeev framework by Barbour and Phillips [13]. A rather transparent derivation, based on the AGS 
equations, and thus more closely related to the above argumentation, was performed by Gibson and Lehman [14. It is, in fact, their approach which has been taken over in subsequent, more sophisticated applications or extensions of momentum-space techniques in photonuclear few-body physics. Only in parenthesis we mention that a derivation, which exhibits in great detail the structural equivalences pointed out above, is found in 15.

In $E 1$ approximation the electromagnetic operator $H_{\mathrm{em}}$ in the above three-nucleon relations is given by

$$
H_{E 1}^{\prime}=-\frac{e}{m} \sum_{j=1}^{3} \frac{1+\tau_{z}^{j}}{2} \hat{\epsilon} \cdot \vec{P}_{j}
$$

With inclusion of meson exchange currents (MEC) we have, according to Siegert's theorem, an expression analogous to (1.7),

$$
M(\vec{q})=-i e\left(E_{f}-E_{i}\right)^{(-)}\left\langle\vec{q} ; \psi_{d}\left|\sum_{j=1}^{3} \frac{1+\tau_{z}^{j}}{2} \hat{\epsilon} \cdot \vec{X}_{j}\right| \psi_{t}\right\rangle .
$$

Here $\vec{P}_{j}, \vec{X}_{j}$ and $\tau_{z}^{j}$ are the momentum and position operators, and the isospin Pauli matrices of the three nucleons involved. As in (1.8) the difference of the final energy $E_{f}=q^{2} / 2 M+E_{d}$ and the initial triton energy $E_{i}=E_{t}$ is just the energy of the incident photon, $\left(E_{f}-E_{i}\right)=E_{\gamma}$. Let us mention that for $s$-wave potentials only that part of the $E 1$ electromagnetic operator which acts between the fragments survives, while, due to its $p$-wave structure, the contribution to $H_{E 1}$ acting within the deuteron vanishes. The electromagnetic operator then reduces to

$$
H_{E 1}=-i e\left(E_{f}-E_{i}\right)\left(\frac{\tau_{z}^{1}+\tau_{z}^{2}}{2}-\tau_{z}^{3}\right) \hat{\epsilon} \cdot \vec{R}_{3}
$$

where $\vec{R}_{3}$ denotes the relative position operator between the elementary particle 3 and the bound state of particles 1 and 2. Although, in general, higher partial waves are quite relevant in realistic potentials, (1.22) remains the dominant part of $H_{\mathrm{em}}$ also in such cases.

In the following considerations of the total cross section, the $E 1$ contributions are sufficient. A considerable influence of the electric quadrupole (E2) contribution, however, shows up in the differential cross section. The corresponding operator is given by

$$
H_{E 2}^{\prime}=-\frac{i e}{2 m} \sum_{j=1}^{3} \frac{1+\tau_{z}^{j}}{2}\left(\hat{\epsilon} \cdot \vec{P}_{j} \vec{k}_{\gamma} \cdot \vec{X}_{j}+\hat{\epsilon} \cdot \vec{X}_{j} \vec{k}_{\gamma} \cdot \vec{P}_{j}\right)
$$

with $\vec{k}_{\gamma}$ the momentum of the incident photon. As in the above relations MEC are included, according to Siegert's theorem, via the replacement

$$
H_{E 2}=\frac{e}{2}\left(E_{f}-E_{i}\right) \sum_{j=1}^{3} \frac{1+\tau_{z}^{j}}{2} \hat{\epsilon} \cdot \vec{X}_{j} \vec{k}_{\gamma} \cdot \vec{X}_{j}
$$




\subsection{Three-nucleon photodisintegration results}

First applications of the above formalism were performed in [13] and [14]. However, only the latter calculations employed the same separable Yamaguchi potential for the determination of $\mathcal{V} \mathcal{G}_{0}$ and the triton wave function in $\mathcal{B}(\vec{q})$. Apart from a physically motivated different choice of the potential parameters in the bound-state case, these calculations, hence, represent the first consistent treatment of the problem within a simple separable potential model. Let us recall in this context that the Yamaguchi potential $V_{l=0}^{s}\left(p^{\prime}, p\right)=\chi\left(p^{\prime}\right) \lambda \chi(p)$, with $\chi(p)=\left(p^{2}+\beta^{2}\right)^{-1}$, is a pure $s$-wave potential, of course with different choices of the parameters $\lambda$ and $\beta$ in the triplet and singlet channels, leading thus to an $s$-wave rank-one separable $T$-matrix.

It is well known that, despite the simplicity of this model, the resulting $n$ - $d$ cross sections reflect essential aspects of the three-nucleon collision problem fairly well, and the same holds true for the corresponding photodisintegration results of [13 and, in particular, of [14]. The main observation made already in these early solutions of the full equations (1.15) is an about $25 \%$ enhancement of the cross section in the low-energy peak region as compared to the Born approximation. This feature appears to be independent of the potential employed [10]. Figure 3 shows this enhancement for the Paris potential. In view of the fact that the outgoing deuteron is a loosely bound, easily polarizable system, such a strong FSI effect is not surprising, while in the elementary-particle NN collision it was found to be negligible (see Sec. 1.1). Even more pronounced (about a factor of 1.7) is the effect of meson exchange currents. It is also much stronger than in the two-body case, an observation easily referred to the higher number of nucleons involved.

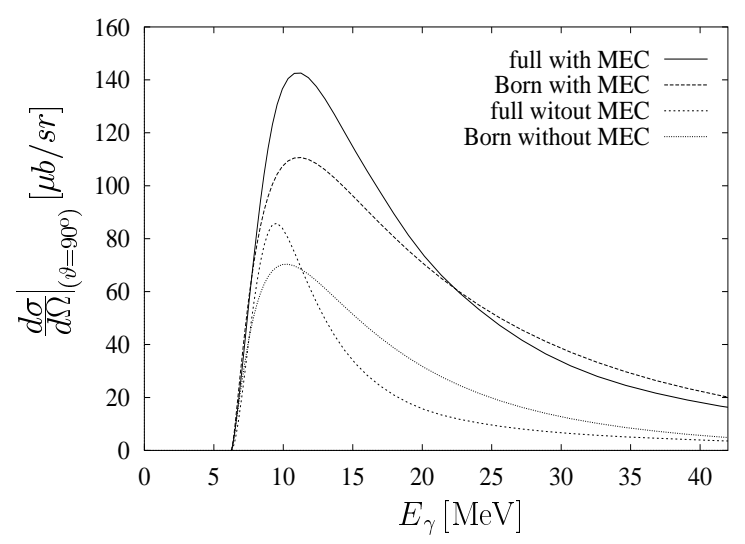

Figure 3. Role of meson exchange currents in the full solution and in Born approximation for the Paris potential $\left(j \leq 1^{+}\right)$.

The transition operator $U_{0 \alpha}$ in the $n d \rightarrow n n p$ break-up amplitude $\left\langle\vec{q}^{\prime}, \vec{p}^{\prime}\left|U_{0 \alpha}\right| \psi_{d}\right\rangle|\vec{q}\rangle$ is obtained by putting $\beta=0$ in the AGS equations (1.10) [9]. This, however, means that it appears as a sum over the rearrangement operators $U_{\beta \alpha}$, and the same holds true for the corresponding amplitudes in the effective two-body formulation, both of the purely nuclear and the corresponding photonuclear processes. For details concerning the $\gamma t \rightarrow n n p$ formalism we refer to [16]. Calculations along this line have been performed exclusively 
for the Yamaguchi potential in [13] and [16]. Again the more consistent calculations of 16 are in better agreement with experiment, although clear discrepancies are also evident. This calls for a repetition of these calculations with more realistic potentials.

\subsection{Potential dependence}

One of the main questions in few-body physics is the sensitivity of relevant observables to the underlying two-body potentials. Due to the fact that the photodisintegration amplitudes contain both the three-body bound and continuum states, these processes are expected to be a good testing ground in this respect. It, in fact, turns out that there are considerable differences between the photonuclear results obtained for the Yamaguchi, Malfliet- Tjon, Paris, Bonn $A$ and Bonn $B$ potentials.

To represent these potentials or the corresponding $T$-matrices in separable form, various techniques have been developed and tested in the purely nuclear case. In the photodisintegration problem it were essentially the $W$-matrix method [17] and the Ernst-Shakin-Thaler (EST) expansion 18 which have been employed within the above momentum-space formalism. The $W$-matrix method is characterized by a particularly simple separable approximation of the $T$-matrix, which preserves all its main analytical and unitary properties. The EST expansions of the Paris, Bonn $A$ and $B$ potentials developed by the Graz group (PEST, BAEST and BBEST) [19,20], have led to the first fully reliable realistic results in the three-nucleon problem [21,22]. In the following applications to photoprocess an improved parametrization by Haidenbauer is used [23].

Fig. 1 gives a good indication for the agreement between both techniques, although for a somewhat limited positive-parity two-body input $\left(j \leq 1^{+}\right)$. The results given below for the Malfliet-Tjon potential are based on the $W$-matrix method. For the Paris and Bonn potentials, $W$-matrix results have also been published [10]. The number of subsystem partial waves employed in these calculations, however, turned out to be too small. We therefore present, instead of these previous results, most recent fully converged calculations [24,25] for the PEST, BAEST, and BBEST potentials.

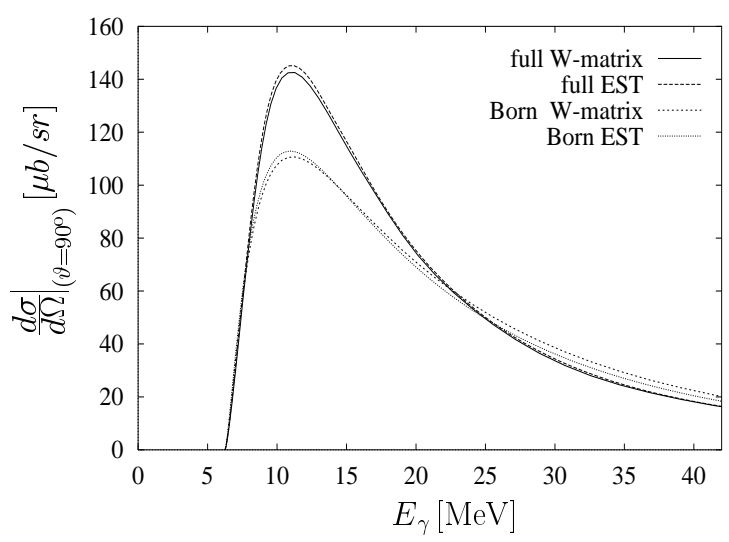

Figure 4. Comparison of the cross sections obtained for the Paris potential $\left(j \leq 1^{+}\right)$using the $W$-matrix and the EST method.

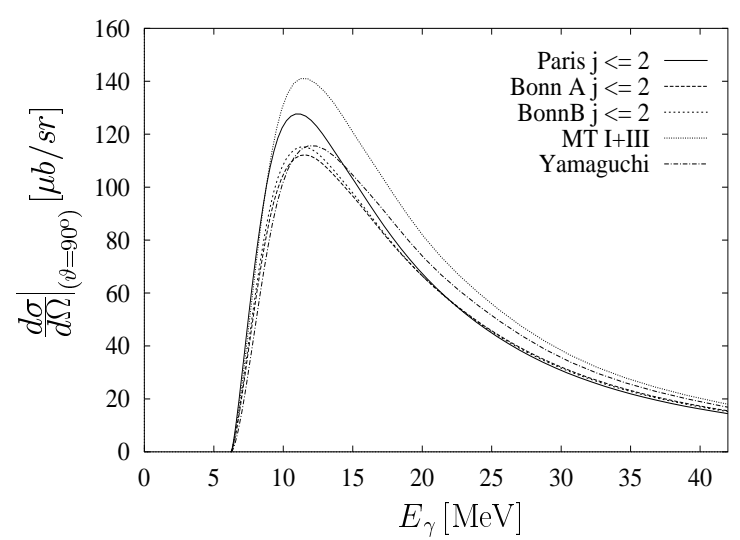

Figure 5. Cross sections for different potentials 
According to Fig. 5, there is a remarkable potential dependence in the peak region, which appears to open the possibility of testing the potentials employed. Unfortunately, as seen in Figs. 668, just in this sensitive region the experimental errors do not allow for a real judgment. This situation definitely calls for new, more accurate measurements.

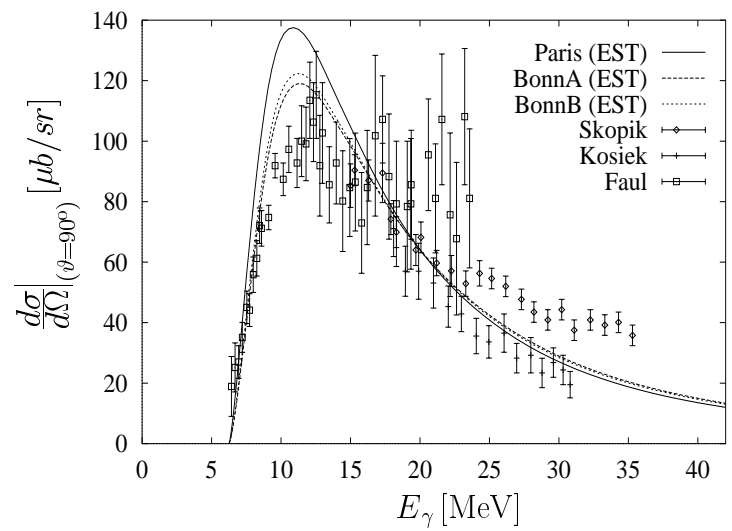

Figure 6. Differential cross section at $\vartheta=90^{0}$ for $\gamma+{ }^{3} \mathrm{H} \rightarrow n+d$ obtained for the PEST, BAEST and BBEST $\left(j \leq 1^{+}\right)$ potentials. The data are from 2628 .

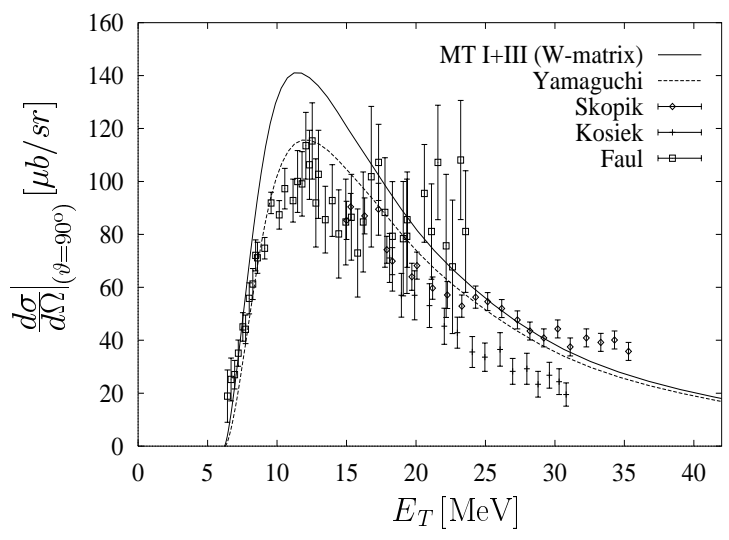

Figure 8. Same as Fig. 6 but for the MT I+III and Yamaguchi potentials.

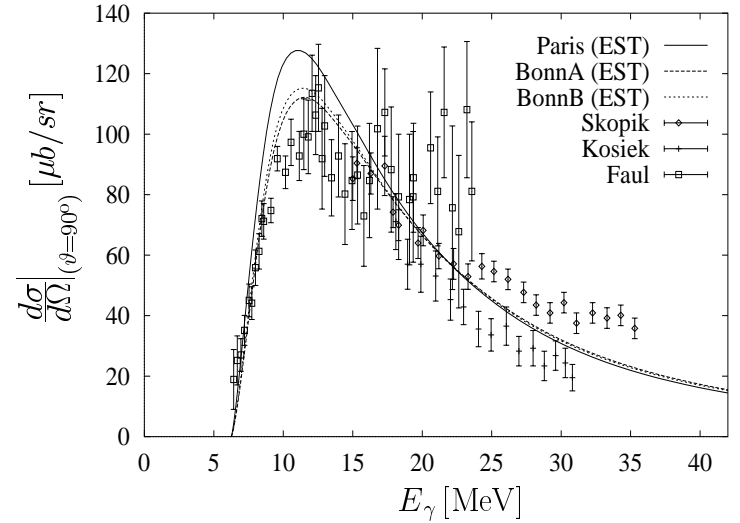

Figure 7. Same as Fig. 6 but with $(j \leq 2)$ contributions in the potentials.

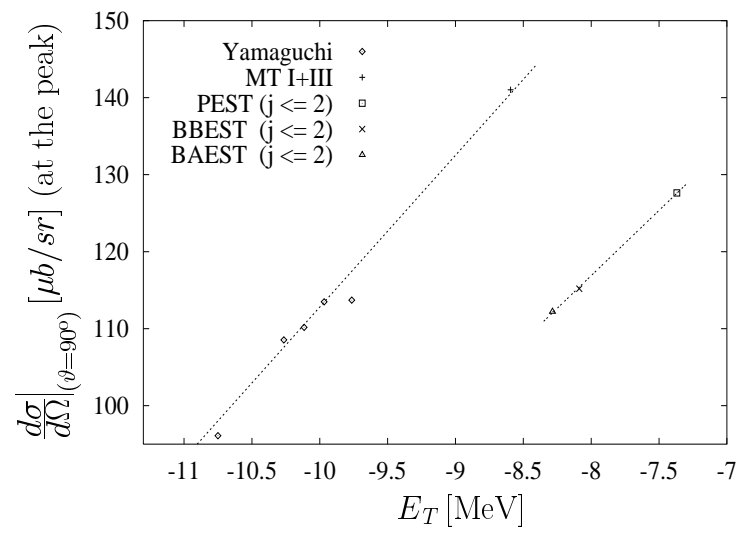

Figure 9. Correlation of the peak heights and the triton binding energies for different potentials.

What has to be asked for is, of course, whether this potential dependence is correlated with other three-body observables. Fig. 9 shows the peak heights of the cross sections versus the corresponding triton binding energies. In this plot the results for Yamaguchi potentials with different parameters and for the Malfliet-Tjon (MT I+III) potential lie on one straight line, the results for the realistic Paris, Bonn $A$ and Bonn $B$ potentials on another, however parallel, line. This strict separation of the two groups of potentials 
is not really a statement about "realistic" or "non-realistic", but is due to the fact that Yamaguchi and Malfliet-Tjon are $s$-wave potentials, while the other ones contain $p$-wave components. In fact, switching on or off parts of these components, one can shift the realistic line towards the Yamaguchi-MT line [24,25]. In any case, the correlation exhibited in Fig. 9 is one of the typical features of few-body physics, as known from the Phillips [29] or Tjon [30] plots.

At higher energies, i.e., above the peak region, the differences between the cross sections for the Yamaguchi and MT potentials, and between the ones for the realistic potentials vanish. However, there is a clear discrepancy between both groups of potentials. It should be emphasized that this discrepancy did not show up in our previous calculations [10], due to the incomplete treatment of relative partial waves mentioned above. While in these previous calculations all potentials seemed to favor in this energy region the measurements by Skopik et al. [28] (this is still true for Yamaguchi and MT), just the realistic ones lie now between these data and the ones by Kosiek et al. [26]. This is another point where a distinct difference between potentials with and without $p$-wave contributions shows up. Also in this respect new measurements, hence, appear desirable.

For completeness it should be mentioned that the role of $p$-wave contributions in the two-body input was emphasized already by Fonseca and Lehman [31]. For an investigation of the dependence on the $d$-state probability in the deuteron, with Yamaguchi-type interactions in these higher partial waves, see [32].

\subsection{Radiative capture}

Detailed balance implies that the solutions of (1.15), i.e., the amplitudes for the triton or ${ }^{3} \mathrm{He}$ photodisintegration into two fragments, determine also the inverse processes, the $n-d$ and $p$ - $d$ radiative capture. In the above considerations of the total cross section it was sufficient to take into account only the $E 1$ part of $H_{e m}$. Fig. 10 shows, in fact, that the $E 2$ contribution, which enters the total cross section additively, is negligibly small.

This is by no means the case for the interference term of the big $E 1$ and the small $E 2$ amplitude occurring in the differential cross section. The $E 2$ contribution, hence, is quite relevant, and evidently needed to achieve the remarkable agreement between theory and experiment. Let us mention that the situation is quite different in $n$ - $d$ capture. There, due to isospin factors, the interference term is strongly suppressed, and does not lead to any noticeable correction of the $E 1$ result.

Figure 11 shows the same $p$ - $d$ process, calculated however in plane-wave (Born) approximation. The general features, in particular the $E 2$ effect, are rather similar to the ones of the full calculation. But, due to the neglect of FSI, the curves lie clearly below the data.

In Fig. 12 it is shown that agreement between theory and experiment is only achieved when incorporating the $p$ - and $f$-wave contributions. Their relevance has been pointed out already in the photodisintegration case. To compare the corresponding observations, we have to bear in mind that the energy of $12.1 \mathrm{MeV}$ corresponds to photodisintegration above the peak region. There, we also found a lowering of the cross sections for potentials with $p$-wave contributions which, however, led to results between the sets of data. The excellent agreement between theory and experiment in the present case of radiative capture points at the high accuracy of the experimental data, as well as the reliability of 
the calculations.

In the photodisintegration there was practically no potential dependence at somewhat higher energies, and thus, as expected, the difference between the Bonn $A$, Bonn $B$ and Paris results shown in Fig. 13 is also rather small.

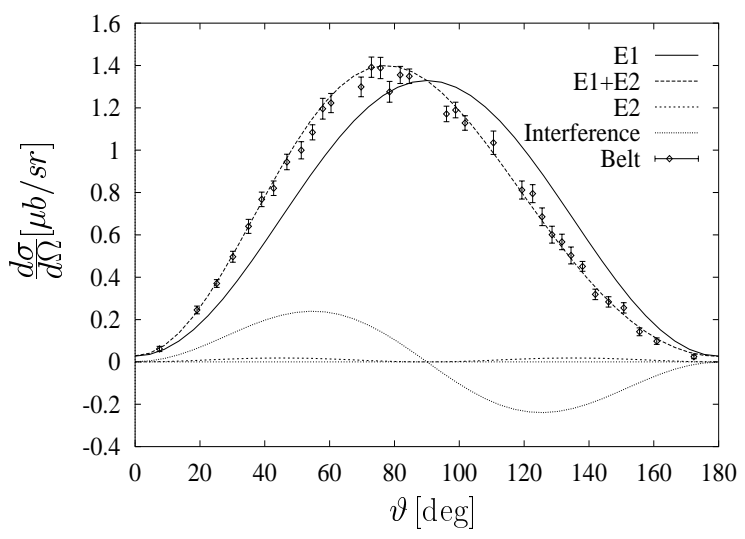

Figure 10. Cross section for $p$ - $d$ capture for the $\operatorname{PEST}(j \leq 2)$ potential at $\mathrm{E}=$ $12.1 \mathrm{MeV}$. The data are from 35

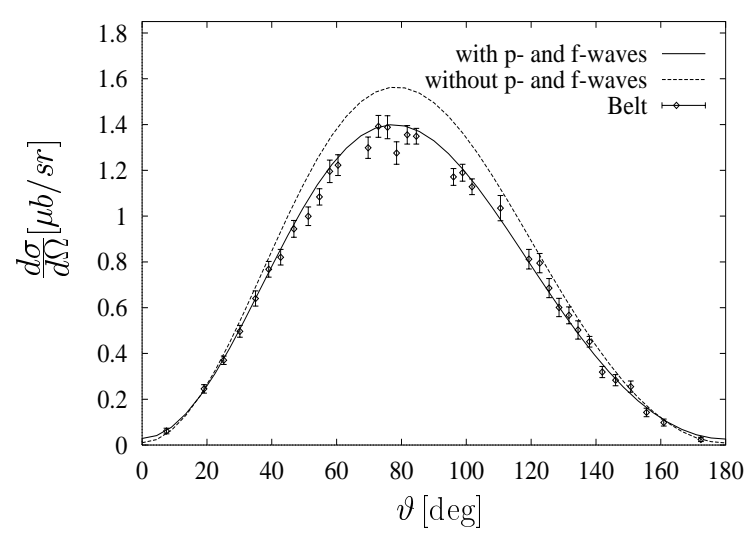

Figure 12. Differential ross section for $p-d$ capture with and without $p$ - and $f$-wave contributions.

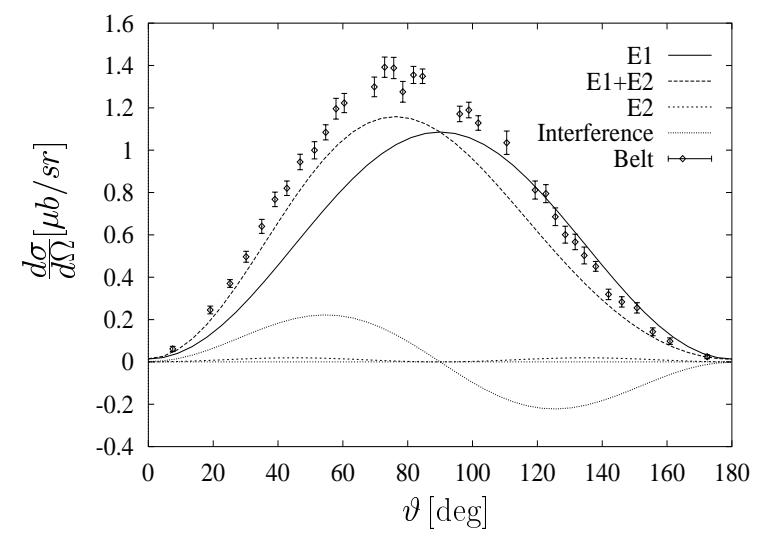

Figure 11. Same as Fig. 10 but in plane wave (Born) approximation.

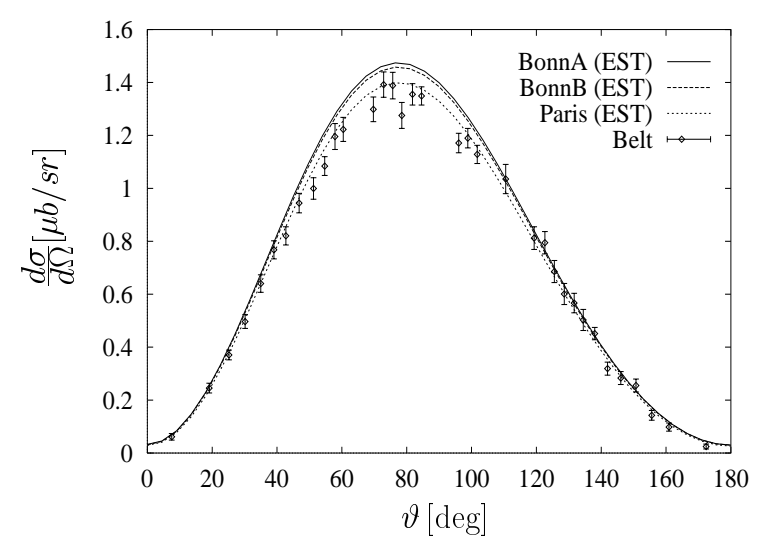

Figure 13. Full calculation of $p-d$ capture at $\mathrm{E}=12.1 \mathrm{MeV}$ for the PEST, BAEST, and $\operatorname{BBEST}$ potentials $(j \leq 2)$.

In our review we tried to give a consistent picture of the influence of FSI, MEC, the potential dependence, the effect of $p$-waves in the interaction, or the $E 2$ contribution in $H_{e m}$, on the cross sections of three-nucleon photodisintegration or radiative capture. It should be mentioned that such questions have been asked also with respect to polarization observables in radiative capture [31 34]. In the calculations by Fonseca and Lehman, e.g., a strong FSI and $p$-wave effect was found for the tensor analyzing power $T_{20}$ and $A_{y y}$. 
Very low energy radiative capture processes are of considerable astrophysical relevance. The $n$ - $d$ radiative capture, which at such energies is almost entirely a magnetic dipole (M1) transition, was studied in plane wave (Born) approximation by Friar et al. [36. In these investigations the authors employed their configuration-space Faddeev calculations of the triton wave function, with inclusion of three-body forces and pion exchange currents. Various trends, e.g. the correlation between cross sections and triton binding energies, and its potential dependence were pointed out.

More recently a rather detailed investigation of such processes has been performed by Viviani et al. [37]. Their calculations employed the very accurate three-nucleon boundand continuum states, obtained in the variational pair-correlated hyperspherical method developed, tested, and applied over years by this group. For a review we refer to the invited talk by Viviani at this conference 38.

\section{FOUR-BODY PROCESSES}

In this section we recall the generalization of the three-body momentum space approach to the four-body case, and review its applications to the photoprocesses $\gamma+{ }^{4} \mathrm{He} \rightarrow n+{ }^{3} \mathrm{He}$ or $p+{ }^{3}$ He. Similarly to the three-body case, we study the influence of FSI, MEC, the potential dependence, or $E 2$ contributions on the corresponding total and differential cross sections.

\subsection{Formalism}

The generalization of the AGS equations (1.10) is the set of $18 \times 18$ operator identities 39

$$
U_{\beta \alpha}^{\sigma \rho}=\left(1-\delta_{\sigma \rho}\right) \delta_{\beta \alpha} G_{0}^{-1} T_{\alpha}^{-1} G_{0}^{-1}+\sum_{\tau \neq \sigma} \sum_{\gamma} U_{\beta \gamma}^{\tau} G_{0} T_{\gamma} G_{0} U_{\gamma \alpha}^{\tau \rho}
$$

for the generalized four-body transition operators $U_{\beta \alpha}^{\sigma \rho}$. As in the AGS equations, this set contains in its kernel the two-body operators $T_{\gamma}=T_{i j}$. But now there occur also the threebody operators $U_{\beta \alpha}^{\tau}$ given by (1.10). The indices $\rho, \sigma, \tau$ denote the three-body subsystems in $(3+1)$ fragmentations or the possible $(2+2)$ fragmentations. As an equation for fourbody quantities it acts on three relative (Jacobi) variables being thus three-dimensional after partial wave decomposition.

Inserting the separable expansion (1.11), its dimension is reduced, as usual, by one. That is, we now end up with an effective two-body equation of exactly the AGS form (1.10),

$$
\mathcal{U}^{\sigma \rho}=\left(1-\delta_{\sigma \rho}\right) \mathcal{G}_{0}^{-1}+\sum_{\tau \neq \sigma} \mathcal{T}^{\tau} \mathcal{G}_{0} \mathcal{U}^{\tau \rho}
$$

As in the genuine three-body case it may be, and has been used as it stands, i.e., as a two-dimensional relation. But again it appears more convenient to further reduce the dimension by expanding also the operator $\mathcal{T}^{\tau}$ into separable terms. This two-step reduction scheme, concerning both the two- and three-body subsystem operators in the four-body equations, provides us then with an effective two-body matrix equation

$$
\mathcal{T}\left(\vec{q}^{\prime}, \vec{q}\right)=\mathcal{V}\left(\vec{q}^{\prime}, \vec{q}\right)+\int d^{3} q^{\prime \prime} \mathcal{V}\left(\vec{q}^{\prime \prime}\right) \mathcal{G}_{0}\left(q^{\prime \prime}\right) \mathcal{T}\left(\vec{q}^{\prime \prime}, \vec{q}\right)
$$


of the LS form (1.3) or (1.12), of course with a much more complicated structure of the potentials $\mathcal{V}$ and Green functions $\mathcal{G}_{0}$. Here, $\vec{q}$ and $\vec{q}^{\prime}$ denote the relative momenta between the clusters in the $(3+1)$ or $(2+2)$ channels.

And, analogously to the steps leading in the two- and three-body cases to the photodisintegration equations (1.6) and (1.15), we end up with their four-body counterpart 40

$$
\mathcal{M}(\vec{q})=\mathcal{B}(\vec{q})+\int d^{3} q^{\prime} \mathcal{V}\left(\vec{q}, \vec{q}^{\prime}\right) \mathcal{G}_{0}\left(\vec{q}^{\prime}\right) \mathcal{M}\left(\vec{q}^{\prime}, \vec{q}\right)
$$

for an off-shell extension of the (properly antisymmetrized) four-body photodisintegration amplitude

$$
M(\vec{q})=2^{(-)}\left\langle\vec{q} ; \psi_{I I I}\left|H_{e m}\right| \psi_{I V}\right\rangle
$$

Again the effective potentials and Green functions can be taken over from the purely nuclear case, i.e., from Eq. (2.3), and the inhomogeneity $\mathcal{B}(\vec{q})$ is an off-shell extension of the plane-wave (Born) approximation of this process,

$$
B(\vec{q})=2\langle\vec{q}|\left\langle\psi_{I I I}\left|H_{e m}\right| \psi_{I V}\right\rangle .
$$

In what follows we show results obtained for the Yamaguchi potential and the semirealistic Malfliet-Tjon (MT I+III) potential. For the treatment of the two-and three-body subsystem operators in the kernel of the four-body equations, the $W$-matrix approximation [17] and the energy dependent pole expansion (EDPE) 41] were chosen. In the pure nuclear case these two approximations have led to rather accurate results. The high accuracy of the $W$-matrix representation in the photonuclear case, moreover, was demonstrated in Sec. 1.4 (see, in particular, Fig. 4). The accuracy of the EDPE is, of course, less evident and involves, in fact, one of the uncertainties of the present four-nucleon momentum-space approach.

The electromagnetic $E 1$ and $E 2$ operators, with and without Siegert replacement, are the ones given in Eqs. (1.20), (1.21), (1.23) and (1.24). The sums runs now, of course, till $j=4$.

\subsection{Results}

Figure 14 shows the total ${ }^{4} \mathrm{He}(\gamma, n){ }^{3} \mathrm{He}$ cross sections for the MT potential. Due to the complicated cut structure of the integral equation kernel, the full calculations could be performed only below the three-fragment break-up threshold. Even more pronounced than in the three-body case (compare Fig. 3) is the difference between the full and the Born, the MEC and the non-MEC results. Note that only with inclusion of these effects the remarkable agreement between theory and experiment is achieved 42.

Fig. 15 shows the corresponding results for the Yamaguchi potential. They lie below the MT curves, in fact too low as compared to the data. This potential dependence resembles completely the one exhibited in the three-body case. Again, a stronger ${ }^{4} \mathrm{He}$ binding (39.1 $\mathrm{MeV}$ for Yamaguchi as compared to $30.1 \mathrm{MeV}$ for MT I+II) leads to a lowering of the cross section in the peak region. 
According to the three-body experience, FSI contributions can be neglected at higher energies. There, as shown in Figs. 16 and 17, also the potential dependence vanishes, and the $E 2$ contributions are small. All these observations are supported by the remarkable agreement with newer experimental data [46]. The two- and three-body experiences, on the other hand, indicate a strong $E 2$ effect in the differential cross sections. And, in fact, the ${ }^{4} \mathrm{He}(\gamma, p){ }^{3} \mathrm{H}$ differential cross sections in Figs. 18 and 19 show E2 corrections similarly to the ones of Figs 2, 10, and 11. In contrast to the three-body case, there remain, however, discrepancies which, according to the experiences gained in this case, are probably to be attributed to the neglect of higher partial waves in the two- and threebody subsystems. An improvement of the EDPE, the incorporation of further multipoles or FSI corrections may also be considered in this context.

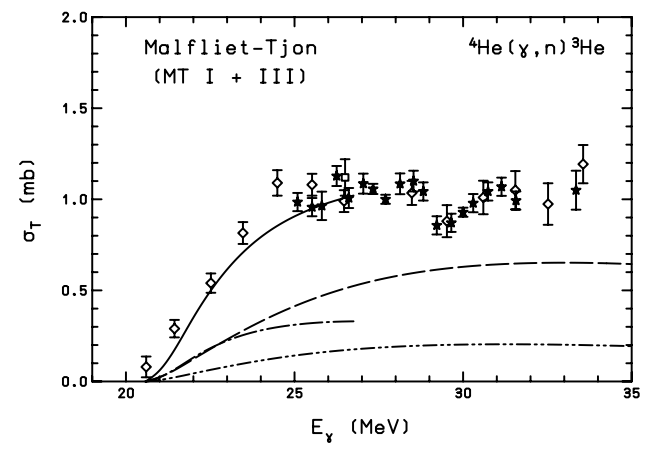

Figure 14. Total cross section for ${ }^{4} \mathrm{He}(\gamma, n){ }^{3} \mathrm{He}$ at low energies: Full solution with exchange currents $(-)$ and without $(-\cdot-)$; plane wave approximation with exchange currents $(--)$ and without $(-\cdots-)$; the data are from 43] $\diamond$, 44 $\star$, and 45 $\square$.

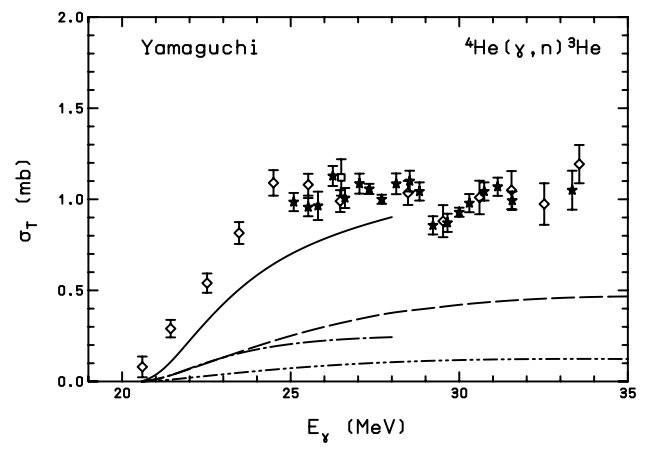

Figure 15. Same as Fig. 14, but with the Yamaguchi potential.

\subsection{Comments and alternative approaches}

The photodisintegration of ${ }^{4} \mathrm{He}$ into $n+{ }^{3} \mathrm{He}$ or $p+{ }^{3} \mathrm{H}$ has for a long time been a rather controversial topic. Early data appeared consistent with the picture of a giant dipole resonance, a feature also supported by shell model [49], and resonating group (RGM) calculations [50] In contrast, applications of the momentum-space integral equation approach indicated from the very beginning a fairly flat, non-resonant behavior [40,51], and at the same time a similar trend was observed also experimentally [43].

The drastic separable approximations of the three-body transition operators in the kernel of the four-body equations, employed in these calculations, have been replaced in the meantime by the more reliable EDPE, and additional new measurements are also available. As seen in Fig. 14, all these developments have confirmed the flat low-energy behavior of the total cross section predicted in 40. and 43.

As mentioned already, the RGM calculations by Wachter et al. [50] reproduced the 


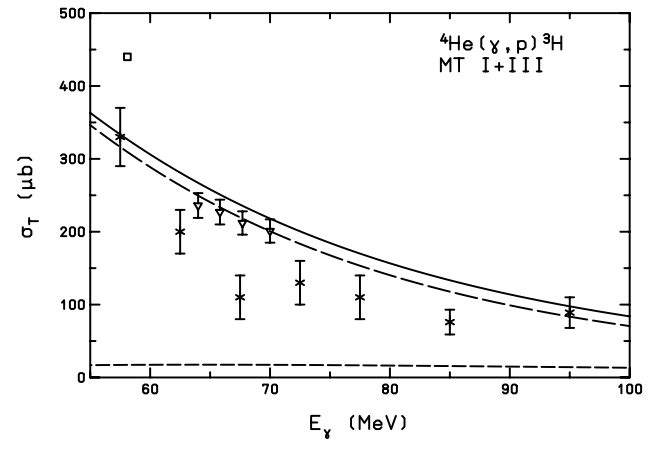

Figure 16. Total cross section for ${ }^{4} \mathrm{He}(\gamma, p){ }^{3} \mathrm{H}$ at higher energies: Plane wave approximation including E1 and E2 $(-)$. Contributions of E1 (- -$)$ and E2 (- - ) ; the data are from 46] $\nabla, 47$ $\times$, and 48 .

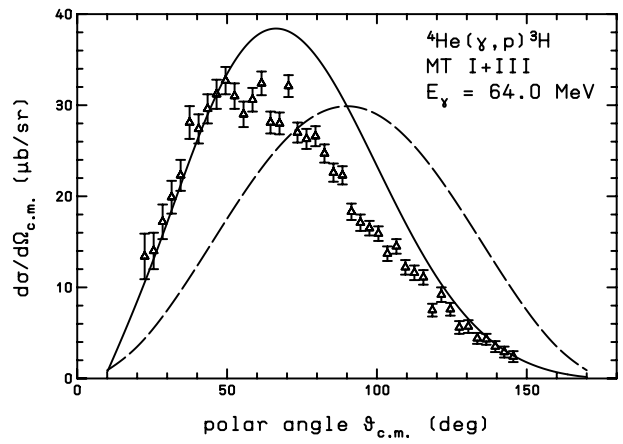

Figure 18. Differential cross section: Plane wave approximation including E1 and E2 (-). Contribution of E1 only $(--)$; the data are from [46].

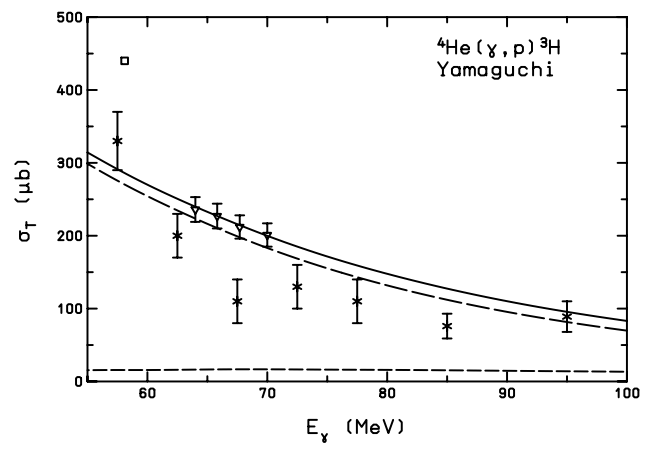

Figure 17. Same as Fig. 16, but with the Yamaguchi potential.

apparently non-existing giant resonance. This is not surprising. Their application of the RGM approach is characterized by a completely unrealistic potential chosen for purely technical reasons. The corresponding binding energies, e.g., differ from the experimental ones by almost a factor of two. To cure this shortcoming, the authors replaced the theoretical by the experimental values in the Siegert factor. This inconsistency allowed them to fit first the giant resonance behavior and then, by going back to the unrealistic theoretical values, also the new flat results [52]. No need to emphasize that this high ambiguity reduces the predictive power of such a method.

The complexity of the full four-body integral equations suggests to look for somewhat less ambitious, but none the less sufficiently reliable models. The approach suggested in 53] is based on writing the full outgoing scattering state as a product

$$
{ }^{(-)}\left\langle\vec{q}^{\prime} ; \psi_{I I I}\right| \simeq{ }^{(-)}\left\langle\vec{q}^{\prime}\right|\left\langle\psi_{I I I}\right|
$$


Here, the two-fragment scattering state ${ }^{(-)}\langle\vec{q}|$ describes the motion of the outgoing nucleon under the influence of an optical potential, adjusted to the $n-{ }^{3} \mathrm{He}$ or $p-{ }^{3} \mathrm{H}$ scattering data. A further simplification consists in calculating the three- and four-body bound states within the integrodifferential approach (IDEA) [54]. The results obtained in this way for the total cross section are in good agreement with those of the full treatment. This model, moreover, allows one immediately go beyond the three-body break-up threshold (compare the comments on Fig. 14). The Coulomb effect, moreover, is easily incorporated.

Note that the determination of the optical potential between the outgoing fragments was based in [53] on a simple analytical expression adjusted to the data. Instead, the more sophisticated Marchenko inversion technique could be employed, as discussed with respect to electrodisintegration in Sec. 3.3.

\section{THREE- AND FOUR-NUCLEON ELECTRODISINTEGRATION}

Our main topic is the photodisintegration or radiative capture of light nuclei. The considerable experimental and theoretical interest devoted to the corresponding electrodisintegration processes, however, suggests to briefly review also methods developed, and results achieved in this field, and to compare them with a recent alternative access to this problem based on Marchenko inversion.

\subsection{Three-nucleon problem}

Early calculations of electron-induced nuclear disintegration processes, with the scattered electron detected in coincidence with the ejected proton ("coincidence cross section"), were performed by Griffy and Oakes [55] using the Irving three-body wave functions or a pole approximation approach. The corresponding results (dashed or dotted lines) are shown in Fig. 20. Few-body techniques were employed by Lehman to obtain more appropriate wave functions. He calculated in this way the two- and three-fragment electrodisintegration of ${ }^{3} \mathrm{H}$ and ${ }^{3} \mathrm{He}$ for the Yamaguchi potential, although without FSI [56].

The Gibson-Lehman approach, i.e., the Faddeev-AGS-type treatment of photodisintegration [14 discussed in Sec. 1.2 was extended to ${ }^{3} \mathrm{H}$ and ${ }^{3} \mathrm{He}$ electrodisintegration by Heimbach et al. [58. Being based on the fairly simple Yamaguchi potential, this procedure takes into account consistently the final-state interaction. Van Meijgaard and Tjon [59 employed the MT I+III potential in unitary pole expansion (UPE), and found a considerable difference between the Yamaguchi and MT results.

More realistic interactions, the Urbana and Argonne potentials, were used in the variational Monto Carlo (VMC) 60 calculations by Schiavilla and Pandharipande 61, while Laget 62 employed a diagrammatic expansion of the amplitudes, taking into account in this way at least part of FSI and MEC.

In a more rigorous approach Ishikawa et at. 63. calculated ${ }^{3} \mathrm{He}$ electrodisintegration by a 34-channel treatment of Faddeev-type two-dimensional equations. The results for the Paris- and Bonn $B$ potentials obtained in this way are shown in Fig. 21.

\subsection{Four-nucleon problem}

In the computationally much more demanding electrodisintegration of ${ }^{4} \mathrm{He}$ into $p+{ }^{3} \mathrm{H}$ or $n+{ }^{3}$ He practically no exact calculations of the final ${ }^{(-)}\left\langle\vec{q}^{\prime} ; \psi_{I I I}\right|$ scattering state, and 


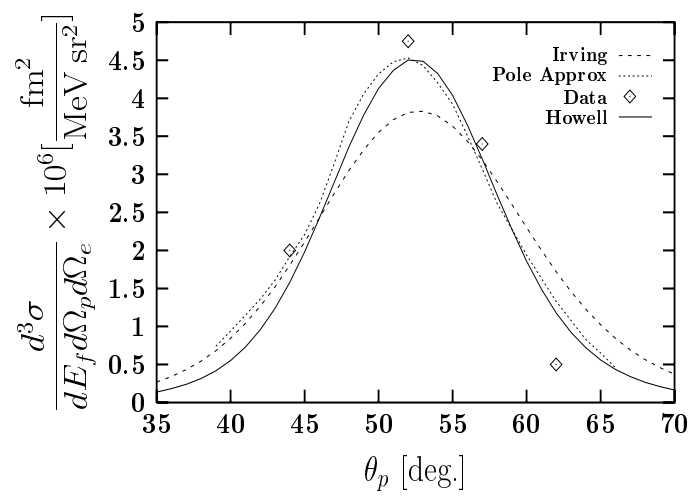

Figure 20. Coincidence cross section for $e+{ }^{3} \mathrm{He} \rightarrow d+p+e^{\prime}$ as a function of the proton scattering angle $\theta_{p}$ for the kinematics of [55]. The reanalyzed data $(\diamond)$ are from [57.

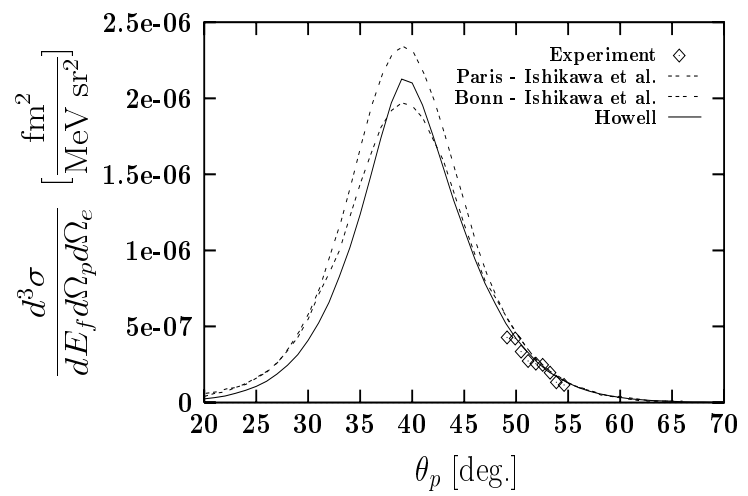

Figure 21. Coincidence cross section for the $\mathrm{C} 2$ kinematics of 63 .

thus of FSI, has been performed. One therefore resorts to the approximation

$$
{ }^{(-)}\left\langle\vec{q}^{\prime} ; \psi_{A-1}\right| \simeq{ }^{(-)}\left\langle\vec{q}^{\prime}\right|\left\langle\psi_{A-1}\right|
$$

traditionally used in nuclear physics. Here, ${ }^{(-)}\left\langle\vec{q}^{\prime}\right|$ represents the scattering state of the elementary particle interacting with the composite particle $\left\langle\psi_{A-1}\right|$ via an appropriately chosen optical potential. A decisive criterion for the quality of this approximation is that it does not violate the orthogonality condition between bound and scattering states. In other words, the condition

$$
{ }^{(-)}\left\langle\vec{q}^{\prime}\right|\left\langle\Psi_{A-1} \mid \Psi_{A}\right\rangle \simeq 0
$$

has to be satisfied with high accuracy. We refer in this context to the investigations by Schiavilla and Pandharipande [61] and to the extension of their above-mentioned threebody calculations to the four-body case [64]. The three-body approach by Laget has also its four-body counterpart 65. The main findings of these investigations were that in certain kinematical regions there is an extreme sensitivity to the nuclear forces and that the different models employed lead to fairly distinct results.

\subsection{Marchenko inversion approach}

The considerable effort required when treating three- and four-body disintegration processes in their full complexity, suggests to look more closely at approximations of the type (3.8). Assuming this approximation to be justified, the main problem consists in constructing the effective interaction between the outgoing fragments in a reliable manner.

L.L. Howell et al. 66, 67 employed for this purpose the Marchenko inversion method. The bound states are calculated by means of the comparatively simple integrodifferential equation approach (IDEA) [54. The, in principle, exact Marchenko treatment requires the knowledge of the high-energy behavior of the nucleon-nucleus phase shifts. The lack of data at such energies allows for various extrapolations leading to different effective 
potentials and thus, via the corresponding scattering states ${ }^{(-)}\langle\vec{q}|$, to different electrodisintegration results. In other words, proceeding in this way provides an appropriate tool for testing the high energy behavior of the phase shifts in a fairly sensitive physical situation.

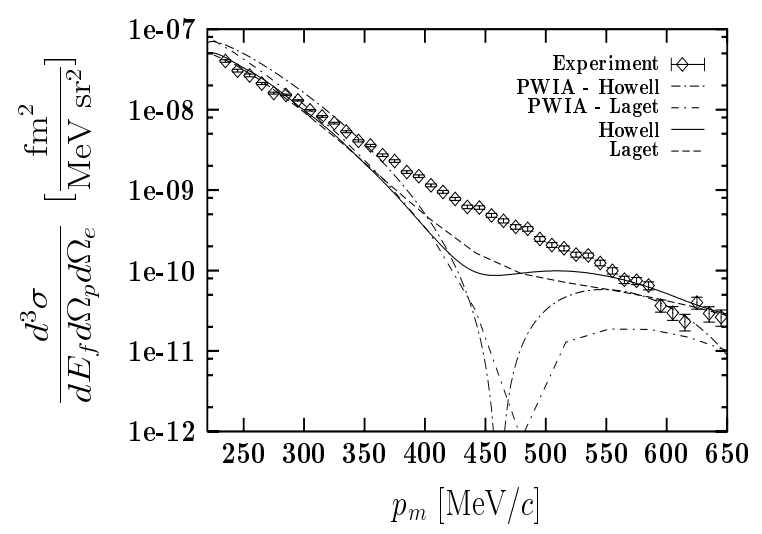

Figure 22. Coincidence cross section for the two-fragment electrodisintegration of ${ }^{4} \mathrm{He}$ as a function of the missing momentum for the kinematics (A,B,C,D) of Ref. 668.

The apparent freedom in extrapolating the phase shifts practically vanishes when imposing the orthogonality condition $(3.9)$ on the resulting wave function ${ }^{(-)}\left\langle\vec{q}^{\prime}\right|$. The solid line in Fig. 20 obtained in this way agrees remarkably well with the pole-approximation results and thus with the data. In Fig. 21 the corresponding solid line lies between the Paris and Bonn results of Ishikawa et al. [63. In other words, this model provides highly reliable results within a comparatively simple model approach, which moreover sheds some light on the high-energy behavior of the nucleon-nucleus phase shifts. It, hence, leads to quite relevant informations concerning the construction of optical potentials.

An example for ${ }^{4} \mathrm{He}$ electrodisintegration at higher energies is shown in Fig. 22 The characteristic dip found in the plane wave (Born) approximation is partly, but not fully, removed when taking into account the FSI by means of Laget's diagrammatic technique [65]. The Marchenko inversion approach by Howell et al. [66, 67] agrees fairly well with the corresponding results. That is, both in the three- and in the four-body case it represents a rather natural, comparatively simple alternative to other methods.

With respect to the remaining discrepancies in the dip region, which are neither cured by the Laget nor the Marchenko inversion procedure, it appears necessary to incorporate, at least approximately, the interplay of the $(3+1)$ and $(2+2)$ amplitudes, which appears quite essential in the fully coupled system of equations. (compare in this context the discussion in Sec. 2.1). Investigations in this direction are in progress.

\section{REFERENCES}

1. H. Arenhövel and M. Sanzone, Photodisintegration of the Deuteron (Few-Body Systems Suppl. 3 (Springer-Verlag, Wien, 1991).

2. A. J. F. Siegert, Phys. Rev. 52, 787 (1937). 
3. H. Arenhövel and M. Schwamb, private communication.

4. C. A. Barnes, J. H. Carver, G. H. Stafford, and D. H. Wilkinson, Phys. Rev. 86, 359 (1952).

5. Y. Birenbaum, S. Kahane, and R. Moreh, Phys. Rev. C 32, 1825 (1985).

6. D. M. Skopik, Y. M. Shin, M. C. Phenneger, and J. J. Murphy, Phys. Rev. C 9, 531 (1974).

7. De Pascale et al., Phys. Lett. 119B, 30 (1982); Phys. Rev. C 32, 1830 (1985).

8. K. H. Krause et al., Physics with MAMI, S. 85 (Institut für Kernphysik/SFB 201, Universität Mainz, 1988).

9. E. O. Alt, P. Grassberger, and W. Sandhas, Nucl. Phys. B2, 167 (1967).

10. W. Schadow and W. Sandhas, Phys. Rev. C 55, 1074 (1997).

11. L. Canton and W. Schadow, Phys. Rev. C 56, 1231 (1997).

12. W. Schadow, W. Sandhas, and J. Haidenbauer, to be published.

13. I. M. Barbour and A. C. Phillips, Phys. Rev. Lett. 19, 1388 (1967).

14. B. F. Gibson and D. R. Lehman, Phys. Rev. C 11, 29 (1975).

15. W. Sandhas, in Few-Body Systems, Suppl. 1 (Springer-Verlag, Wien, 1986).

16. B. F. Gibson and D. R. Lehman, Phys. Rev. C 13, 477 (1976).

17. E. A. Bartnik, H. Haberzettl, and W. Sandhas, Phys. Rev. C 34, 1520 (1986).

18. D. J. Ernst, C. M. Shakin, and R. M. Thaler, Phys. Rev. C 8, 46 (1973); Phys. Rev. C 9, 1780 (1974).

19. J. Haidenbauer and W. Plessas, Phys. Rev. C 30, 1822 (1984); Phys. Rev. C 32, 1424 (1985).

20. J. Haidenbauer, Y. Koike, and W. Plessas, Phys. Rev. C 33, 439 (1986).

21. J. Haidenbauer and Y. Koike, Phys. Rev. C 34, 1187 (1986).

22. Y. Koike, J. Haidenbauer, and W. Plessas, Phys. Rev. C 35, 396 (1987).

23. J. Haidenbauer, private communication.

24. W. Schadow and W. Sandhas, contribution to this conference.

25. W. Schadow and W. Sandhas, to be published.

26. R. Kosiek, D. Müller, and R. Pfeiffer, Phys. Lett. 21, 199 (1966).

27. D. D. Faul, B. L. Berman, P. Meyer, and D. L. Olson, Phys. Rev. Lett. 44, 129 (1980);

Phys. Rev. C 24, 849 (1981).

28. D. M. Skopik, D. H. Beck, J.Asai, and J. J. Murphy II, Phys. Rev. C 24, 1791 (1981).

29. A. C. Phillips, Nucl. Phys. A107, 290 (1968).

30. J. A. Tjon, Phys. Rev. Lett. 56B, 217 (1975).

31. A. C. Fonseca and D. R. Lehman, Phys. Rev. C 48, R503 (1993).

32. A. C. Fonseca and D. R. Lehman, in Few-Body Systems, Suppl. 6 (Springer-Verlag, Wien, 1992).

33. A. C. Fonseca and D. R. Lehman, Phys. Lett. B 267, 159 (1991).

34. S. Ishikawa and T. Sasakawa, Phys. Rev. C 45, R1428 (1992).

35. B. D. Belt, C. R. Bingham, M. L. Halbert, and A. van der Woude, Phys. Rev. Lett. 24, 1120 (1970).

36. J. L. Friar, B. F. Gibson, and G. L. Payne, Phys. Lett. B 251, 11 (1990).

37. M. Viviani, Schiavilla, and A. Kievsky, Phys. Rev. C 54, 534 (1996).

38. M. Viviani, Invited talk presented at this conference.. 
39. P. Grassberger and W. Sandhas, Nucl. Phys. B2, 181 (1967); E. O. Alt, P. Grassberger, and W. Sandhas, Phys. Rev. C 1, 85 (1970) and in Few Particle Problems in the Nuclear Interaction, edited by I. Slaus (North-Holland, Amsterdam, 1972), p. 299; W. Sandhas, in Progress in Particle Physics, edited by P. Urban (Acta Physica Austriaca, Suppl. XIII, 1974), p. 679; Czech. J. of Phys. B25, 251 (1975).

40. A. Casel and W. Sandhas, in Proc. Ninth Int. Conference on the Few-Body Problem, Vol I: Contributed Papers, ed. M.J. Moravcsik (Eugene, Oregon, 1980); see also W. Sandhas: Few-Body Systems Suppl. 1, 64 (1986); W. Böttger, A. Casel, and W. Sandhas, Phys. Lett. 92B, 11 (1980).

41. S. Sofianos, N. McGurk, and H. Fiedeldey, Nucl. Phys. A318, 295 (1979).

42. G. Ellerkmann, W. Sandhas, S. A. Sofianos, and H. Fiedeldey, Phys. Rev. C 53, 2638 (1995).

43. B. Berman et al., Phys. Rev. C 22, 2273 (1980).

44. L. Ward et al., Phys. Rev. C 24, 317 (1981).

45. J. Asai et al.: Few-Body Systems Suppl. 7, 136 (1994).

46. R. Jones et al., Phys. Rev. C 43, 2052 (1991).

47. A. N. Gorbunov, Proc. of the P. N. Lebedev Physics Institute 71, 1 (1976).

48. R. Bernabei et al., Phys. Rev. C 38, 1990 (1988).

49. D. Halderson and R. Philpott, Nucl. Phys. A359, 365 (1981).

50. B. Wachter, T. Mertelmeier, and H. M. Hofmann, Phys. Rev. C 38, 1138 (1988).

51. A. Casel and W. Sandhas, Czech. J. of Phys. B36, 300 (1986).

52. M. Unkelbach and H. M. Hofmann, Nucl. Phys. A549, 550 (1992).

53. S. A. Sofianos, H. Fiedeldey, and W. Sandhas, Phys. Rev. C 48, 2285 (1993).

54. M. Fabre de la Ripelle, H Fiedeldey and S. A. Sofianos, Phys. Rev. C 38, 449 (1988)

55. T. A. Griffy and R. J. Oakes, Phys. Rev. 135, B1161 (1964); Rev. Mod. Phys. 37, 402 (1965).

56. D. R. Lehman, Phys. Rev. Lett. 23, 1339 (1969).

57. B. F. Gibson and G. B. West, Nucl. Phys. B1, 349 (1967).

58. C. R. Heimbach, D. R. Lehman, and J. S. O'Connell, Phys. Rev. C 16, 2135 (1977); Phys. Lett. 66B, 1 (1977).

59. E. van Meijgaard and J. Tjon, Phys. Rev. C 42, 74 (1990); Phys. Rev. C 42, 96 (1990); Phys. Rev. C 45, 1463 (1992).

60. R. Schiavilla, V. Pandharipande, and R. Wiringa, Nucl. Phys. A449, 219 (1986).

61. R. Schiavilla and V. Pandharipande, Phys. Rev. C 36, 2221 (1987).

62. J. M. Laget, Phys. Rev. Lett. 151B, 325 (1985).

63. S. Ishikawa et al., Nuovo Cimento 107, 306 (1994).

64. R. Schiavilla, Phys. Rev. Lett. 67, 835 (1990).

65. J. M. Laget, Nucl. Phys. A579, 333 (1994).

66. L. L. Howell, M. Braun, and S. Sofianos, contribution to this conference.

67. L. L. Howell, M. Braun, S. Sofianos, and W. Sandhas, submitted for publication.

68. J. J. van Leeuwe, Ph. D. thesis (unpublished), Rijks Universiteit Utrecht, 1996. 